\title{
GESTÃO DA INFORMAÇÃO NO SEGMENTO MOVELEIRO NAS REGIÕES NORTE E OESTE DO ESTADO DO PARANÁ
}

\author{
Alessandro Vinicios Schneider*, Roberto Rochadelli**, Marcelo Ioris Koche* \\ *Administrador, M.Sc. - schneideravs@ig.com.br - mkoche@unigran.br \\ **Eng. Florestal, Dr., Depto. de Economia Rural e Extensão, UFPR - nashtell@floresta.ufpr.br
}

Recebido para publicação: 25/08/2005 - Aceito para publicação: 04/12/2006

\begin{abstract}
Resumo
Este trabalho apresenta uma pesquisa-diagnóstico no segmento moveleiro, no que tange à gestão da informação aplicada a sistemas empresariais, para promoção de competitividade e perenidade nos negócios. A pesquisa foi realizada no segmento moveleiro nas regiões norte e oeste do estado do Paraná, especificamente nas cidades de Arapongas, Cascavel e Foz do Iguaçu. Foram pesquisadas 35 empresas. $\mathrm{O}$ estudo foi realizado utilizando-se questionários pré-elaborados. Os dados foram tratados de forma qualitativa e quantitativa. $\mathrm{O}$ estudo revelou que as empresas produzem, na grande maioria, móveis para residências e escritórios, sendo que $81,85 \%$ da produção é destinada para o mercado interno, e $18,15 \%$ para a exportação. As informações geradas no departamento de produção representam, para $71,42 \%$ dos empresários, fator de prioridade para a competitividade nos negócios. As micro e pequenas empresas são menos avançadas tecnologicamente. Os principais controles sobre as informações se apresentam no departamento de produção. Já as empresas de porte médio estão em constante aprimoramento dos sistemas e dos processos de gestão da informação, devido a níveis maiores de investimentos, proporcionando um maior controle das informações geradas tanto nas atividades-fim como também nas atividades-meio, oportunizando uma maior competitividade nos negócios, quando comparadas com as micro e pequenas empresas do segmento.

Palavras-chave: Gestão da Informação; segmento moveleiro; sistemas empresariais; competitividade.
\end{abstract}

\begin{abstract}
Management of the information on the furniture segment in the north and west regions of Paraná state. This paper presents a diagnostic research on the furniture segment, as far as management of the information is concerned which is used in system companies, to promote the competitiveness and perennial in business. The research was done in the north and west regions of Paraná state, specifically in the following cities: Arapongas, Cascavel e Foz do Iguaçu. It was researched 35 companies. The study was done through early-elaborated questionnaires. The data were analyzed in a qualitative and quantitative way. The study showed that most companies produce furniture for houses and offices, and $81,85 \%$ of the production is headed on the internal market, and $18,15 \%$ is for exportation. The information generated in the department of production represent, to $71,42 \%$ of the managers, a factor of priority to the competitiveness in business. The micro and small companies are less advanced technologically. The main controls about the information are noticed in the department of production. But the medium companies are continuously improving their systems and the processes of management of the information because of the higher levels of investments, presenting a higher control of the information generated in the end activities as well in the mid ones, giving the chance of a higher competitiveness in business, when they are compared to the micro and small companies of the same segment.

Keywords: Management of the information; furniture segment; system companies; competitiveness.
\end{abstract}

\section{INTRODUÇÃO}

Embora os dados sejam antigos, visto que datam do último Censo Industrial do Instituto Brasileiro de Geografia e Estatística (IBGE), realizado há 16 anos, ainda se afirma que a indústria brasileira de móveis é constituída por aproximadamente 13.500 micro, pequenas e médias empresas, que empregam cerca de 185 mil pessoas. No entanto, entre empresas formais e informais, existem atualmente 
no país mais de 50 mil unidades produtoras de móveis. São empresas familiares tradicionais e, na grande maioria, de capital inteiramente nacional. Recentemente, em alguns segmentos específicos, como o de móveis para escritório, ocorreu a entrada de empresas estrangeiras.

Dentre os fatores positivos que têm marcado o desenvolvimento do setor de móveis na última década, podem ser destacadas a abertura da economia e a ampliação do mercado interno, que, juntamente com a redução da inflação e de seus custos indiretos, têm introduzido novos consumidores, antes excluídos do mercado.

Nos últimos anos, com o aumento havido nas exportações, a indústria aprimorou sua capacidade de produção e apurou significativamente a qualidade de seus produtos, sem que isso significasse aumento dos lucros na mesma proporção. A indústria está investindo atualmente em modernização da tecnologia e na adaptação do design, visando atender aos consumidores de países europeus, especialmente do Reino Unido, e dos Estados Unidos.

Alguns clusters, produtores de móveis no Brasil, vêm apresentando crescimento considerável nos últimos anos, sendo alvo da atenção de diversos segmentos da área governamental em virtude de suas possibilidades, tanto em termos de geração de divisas, proporcionando aumentos substanciais nos volumes exportados, como também de criação de novos empregos e de desconcentração regional.

Esse segmento é bastante competitivo, em razão da disponibilidade de matéria-prima e mão-deobra e da experiência acumulada nos pólos existentes nas regiões Sul e Sudeste.

Com a crescente concorrência global, as empresas terão cada vez mais necessidade de informação, tanto do mercado quanto a respeito dos planos e intenções de consumidores e dos competidores. Essa mesma informação propicia à empresa um conhecimento de sua estrutura de negócios, facilitando o planejamento, a organização, a gestão e o controle dos processos. Assim, a informação servirá como parâmetro, proporcionando alternativas de tomadas de decisão. A dificuldade principal do gestor é determinar, dentre as inúmeras fontes de informação disponíveis dentro e fora da empresa, quais são as relevantes para a execução de seu trabalho.

\section{Objetivo}

Este trabalho objetivou uma pesquisa-diagnóstico no segmento moveleiro no que se refere à gestão da informação aplicada a sistemas empresariais para promoção de competitividade e perenidade nos negócios.

\section{REVISÃO BIBLIOGRÁFICA}

\section{Panorama do segmento moveleiro}

Para Oliveira (1983), a maioria das empresas ligadas ao setor florestal do estado do Paraná é de pequeno e médio porte, encontrando-se com deficiência de informações inerentes ao setor florestal, em face do desconhecimento parcial ou total das fontes geradoras de informações florestais.

$\mathrm{Na}$ análise de Gorini (1998), a introdução de equipamentos automatizados, novas técnicas de gestão empresarial e novas matérias-primas concorreram para o incremento da produtividade na indústria de móveis e para a flexibilização dos processos de produção, os quais passaram a ser produzidos em maiores escalas, perdendo seu caráter artesanal.

Segundo Moraes (2002), a inserção da indústria brasileira de móveis no mercado mundial, ou seja, as exportações nacionais de móveis, sofreu poucas restrições tarifárias por parte de seus parceiros comerciais, e a parcela ocupada pelo Brasil no mercado externo é decorrente da falta de competitividade do produto nacional.

Analisando o desempenho da indústria brasileira de móveis, Coelho-Fuentes e Berger (2004) concluíram que $90 \%$ do crescimento desse setor foram determinados pela ação de fatores internos que afetaram a competitividade internacional do país, tais como a política cambial, o custo de produção, as estratégias competitivas das empresas e a comercialização, entre outros.

\section{Conceitos de sistema de informação}

Sistema de informação pode ser definido como um conjunto de relatórios de determinados sistemas ou unidades departamentais, entregues e circulados dentro da empresa, para uso dos componentes da organização. Pode, também, ser definido como o processo de transformação de dados em 
informações, que são utilizadas na estrutura decisória da empresa e proporcionam a sustentação administrativa, visando à otimização dos resultados esperados (REZENDE; ABREU, 2001a).

Segundo Stair (1998a), sistema de informação é uma série de elementos ou componentes interrelacionados que coletam (entradas), manipulam e armazenam (processo), disseminam (saídas) os dados e informações e fornecem um mecanismo de feedback.

Um sistema de informações eficiente pode ter um grande impacto na estratégia corporativa e no sucesso da empresa. Esse impacto pode beneficiar a empresa, os clientes e qualquer indivíduo ou grupo que interagir com os sistemas de informação (OLIVEIRA, 2001a). Entre os benefícios, podem-se destacar:

- Suporte à tomada de decisão.

- Valor agregado ao produto.

- Melhor serviço e vantagem competitiva.

- Produtos de melhor qualidade.

- Oportunidades de negócios e aumento da rentabilidade.

- Mais segurança nas informações, menos erros, mais precisão.

- Redução de custos e desperdícios.

- Aperfeiçoamento nos sistemas, eficiência, eficácia, efetividade, produtividade.

A informação e o conhecimento serão os diferenciais das empresas e dos profissionais que pretendem destacar-se no mercado, efetivar a perenidade, a sobrevivência, a competitividade e a inteligência empresarial. Assim, a informação deve ser considerada como o diferencial de negócio, quando proporciona alternativas de lucratividade e retornos profícuos para a empresa, seja sedimentando atuações, seja implementando os atuais negócios ou, ainda, criando novas oportunidades de negócios (REZENDE; ABREU, 2001b).

Stair (1998b) comenta que o uso estratégico dos sistemas de informação constitui uma vantagem competitiva e que é um benefício muito importante e de longo prazo para uma empresa. Dessa maneira, o desenvolvimento e a manutenção da vantagem competitiva dependem do processo de valor adicionado oferecido pela empresa.

As empresas que detiverem, organizarem, dominarem e valorizarem mais as informações e o conhecimento do ambiente (interno e externo) em que estiverem envolvidas terão mais condições de competitividade nos negócios (REZENDE; ABREU, 2001c).

Segundo Rezende e Abreu (2001d), os níveis da informação e de decisão empresarial obedecem à hierarquia padrão existente na maioria das empresas, também chamada de pirâmide empresarial, e são conhecidos como estratégico, tático e operacional. O tipo de decisão que é tomada em cada nível requer diferente grau de agregação da informação, e os diferentes níveis de decisão requerem diferentes informações em seus diversos tipos de produtos externados, tais como telas, relatórios, etc.

Para Oliveira (2001b), os subsistemas de informação devem estar compatibilizados com a estrutura de autoridades e de responsabilidades pela execução das atividades estabelecidas pela empresa. Informações destinadas a formular os planos, executar as tarefas e avaliar os desempenhos devem ser estruturadas, quanto ao conteúdo, forma e periodicidade, de acordo com os objetivos das unidades organizacionais, para as pessoas certas em tempo hábil.

Segundo o mesmo autor, a razão básica de se considerar os níveis de influência é que cada um deles proporciona informações diferentes, com amplitude de influência sobre o sistema de informação como um todo, podendo gerar interligações entre os níveis.

O nível estratégico de influência considera a estrutura organizacional de toda a empresa e a melhor interação desta com o ambiente. A informação gerada é de caráter amplo, contemplando a empresa em sua totalidade, ou seja, ambiente externo e interno.

As decisões dão-se no alto escalão da empresa e geram atos cujos efeitos são duradouros e mais difíceis de inverter. Com relação aos níveis hierárquicos, no nível estratégico estão o presidente, os diretores, os sócios, todos chamados de alta administração. Para Oliveira (2001c), nesse nível de influência ocorre uma interação entre as informações do ambiente empresarial (fora da empresa) e as informações internas da empresa.

O nível tático de influência considera determinado conjunto de aspectos homogêneos da estrutura organizacional da empresa. O nível de informação é em grupo (agrupada ou sintetizada), 
contemplando a junção de determinadas informações de uma unidade departamental. As decisões táticas dão-se nos escalões intermediários e geram atos de efeito mais curto e de menos impacto no funcionamento estratégico da empresa. Segundo o mesmo autor, nesse nível de influência ocorre uma aglutinação de informações de uma área de resultado e não da empresa como um todo. Com relação aos níveis hierárquicos, no nível tático estão os gestores de nível médio, ou seja, as gerências, as chefias, os supervisores, em suas respectivas unidades departamentais.

O planejamento operacional pode ser considerado como a formalização de processos, principalmente por meio de documentos escritos, das metodologias de desenvolvimento, das respectivas normas e implementações estabelecidas (OLIVEIRA, 2001d). Além disso, cria condições para a adequação de trabalhos diários da empresa em que o nível operacional de influência considera uma parte específica da estrutura organizacional. O nível de informação é detalhado (analítico), contemplando os pormenores específicos de um dado, de uma tarefa ou atividade.

\section{Gestão da tecnologia da informação}

Tecnologia da informação pode ser todo e qualquer dispositivo que tenha capacidade para tratar dados e/ou informações, tanto de forma sistêmica como empírica, quer esteja aplicada ao produto, quer esteja aplicada ao processo (CRUZ, 1998).

Complementando esses conceitos, a tecnologia da informação está fundamentada nos seguintes componentes:

- Hardware e seus dispositivos e periféricos.

- Software e seus recursos.

- Sistemas de telecomunicações.

- Gestão de dados e informações.

Todos esses componentes interagem e necessitam do componente fundamental, que é o recurso humano. Embora conceitualmente esse componente não faça parte da tecnologia da informação, sem ele essa tecnologia não teria funcionalidade e utilidade (STAIR, 1998c).

$\mathrm{Na}$ maioria das empresas, a tecnologia da informação tem dado excessiva atenção para as técnicas aplicadas à informática, tal como hardware, software e seus periféricos. Gearalmente, se esquecem de sua principal finalidade e utilidade, que é o desenvolvimento e a melhoria dos sistemas de informação, para auxiliar a empresa em seus negócios, processos e atividades (REZENDE; ABREU, 2001d).

\section{MATERIAL E MÉTODOS}

A pesquisa foi realizada no segmento moveleiro do Paraná, em empresas das regiões norte e oeste do estado, especificamente nas cidades de Arapongas, Cascavel e Foz do Iguaçu. Os dados primários desta pesquisa foram obtidos por meio de questionário estruturado, em um total de 35 . As perguntas foram apresentadas de maneira organizada, com todos os entrevistados respondendo as mesmas perguntas e na mesma ordem. Dessa maneira, objetivou-se a padronização das questões, permitindo um maior grau de comparação entre os dados obtidos. Os dados foram tratados de forma descritiva e interpretados à luz do referencial teórico. A análise desses dados teve caráter quantitativo e qualitativo, sendo utilizados instrumentos estatísticos, principalmente as médias, porcentagens e somatórias das respostas dos empresários.

\section{RESULTADOS E DISCUSSÕES}

O universo pesquisado se constituiu de micro, pequenas e médias empresas, com uma média de 30,9 funcionários e amplitude de 4 a 210 funcionários.

As micro e pequenas empresas com até 19 empregados representam em torno de $65 \%$ do total de estabelecimentos registrados e $19 \%$ do emprego total. Já as empresas de porte médio, entre 20 e 500 empregados, representam $35 \%$ do total dos estabelecimentos e $81 \%$ do emprego total.

O padrão identificado na pesquisa está em conformidade com o padrão brasileiro e mundial, sendo, na grande maioria, empresas de pequeno porte e tradicionalmente familiar, com capital inteiramente nacional. 
As empresas produzem, na grande maioria, móveis para residências e para escritórios, sendo que $81,85 \%$ da produção é destinada para o mercado interno e 18,15 \% destinada para a exportação. Essa característica está diretamente ligada ao tamanho das empresas, sendo que as micro e pequenas tendem, na grande maioria, a suprir a demanda do mercado regional onde estão instaladas.

O produto é composto por $78,57 \%$ de insumos da base florestal e o restante por outros tipos de materiais, como ferro, alumínio, plástico, etc.

No que se refere a um departamento específico de tecnologia da informação (TI), apenas cinco (5) empresas o detêm, as quais representam 14,28 \% do universo abordado. São empresas de porte médio, que contam com mais de 100 funcionários, demonstrando uma estreita relação com as empresas $(18,15$ $\%$ que destinam os seus produtos ao mercado externo. As outras 30 empresas, 85,72 \%, não contam com esse departamento, pertencendo ao grupo das micro e pequenas empresas.

Com referência à estrutura organizacional, todas possuem o departamento de produção, 51,42 \% das empresas possuem departamento financeiro, $48,57 \%$ possuem departamento comercial ou de marketing, $28,57 \%$ possuem departamento de materiais ou logístico, $17,14 \%$ possuem departamento de recursos humanos e apenas $8,57 \%$ possuem departamento jurídico-legal.

As informações geradas no departamento de produção representam, para $71,42 \%$ dos empresários, fator de prioridade para a competitividade nos negócios. Em ordem de prioridade, vem o departamento financeiro, o comercial ou de marketing, o de materiais ou de logística e, com menor importância, as informações geradas nos departamentos de recursos humanos e jurídico-legal.

Todas as empresas possuem as informações geradas pelo departamento de produção e as consideram prioritárias para a promoção de vantagem competitiva nos negócios. As ferramentas de gestão de pessoas são pouco utilizadas pelas micro e pequenas empresas, como também os aspectos jurídicos, ambientais e legais são utilizados como instrumentos reativos e não pró-ativos. $\mathrm{O}$ departamento de recursos humanos e o departamento jurídico-legal se apresentam somente nas empresas de porte médio, nas quais a estrutura organizacional e as técnicas de gestão são mais aperfeiçoadas, fatores esses que demonstram uma estreita relação entre o mercado de atuação e as técnicas de gestão dessas empresas.

As micro e pequenas empresas são menos avançadas tecnologicamente, em decorrência de um menor nível de investimento. Os principais controles sobre as informações se apresentam nas atividadesfim e não nas atividades-meio.

As empresas de porte médio são mais avançadas tecnologicamente, devido a um maior nível de investimento em todas as áreas funcionais ou departamentos. Essas empresas detêm um nível maior de controle das informações geradas na empresa como um todo. Estão em constante aprimoramento dos sistemas de informação e dos processos de gestão da informação, oportunizando uma maior promoção da competitividade nos negócios.

\section{CONCLUSÃO}

Ao longo das últimas décadas, ocorreram processos de implantação de equipamentos automatizados e novas matérias-primas, que colaboraram para o incremento da produtividade na indústria de móveis e para a flexibilização dos processos de produção, os quais passaram a produzir em maiores escalas. No que se refere à gestão da informação, o nível de investimentos não ocorreu na mesma proporção, fato que pode ser determinado pelo tamanho das empresas, suas estruturas organizacionais e as técnicas de gestão aplicadas. Em sua grande maioria, são micro e pequenas empresas e tradicionalmente familiares, nas quais a gestão da informação não é totalmente automatizada, devido aos baixos níveis de investimentos nessa área. Os controles dão prioridade aos departamentos de produção, financeiro e comercial ou de marketing, ou seja, nas atividades-fim. Já as empresas de porte médio estão em constante aprimoramento dos sistemas e dos processos de gestão da informação, devido a níveis maiores de investimentos, proporcionando um maior controle das informações geradas tanto nas atividades-fim como também nas atividades-meio, oportunizando uma maior competitividade nos negócios, quando comparadas com as micro e pequenas empresas do segmento.

As micro e pequenas empresas conseguem perceber as vantagens que a gestão da informação pode proporcionar à promoção da competitividade nos negócios, mas devido ao alto custo da tecnologia disponível no mercado, utilizam ferramentas pouco aprimoradas em seu nível de conhecimento.

Independentemente do seu tamanho, toda empresa deve promover mecanismos de controle da informação, pois o gestor necessita de suporte técnico estruturado para qualquer tomada de decisão. 


\section{REFERÊNCIAS}

BUENO, S. Minidicionário da língua portuguesa. São Paulo: FTD, 2000.

COELHO-FUENTES, M. H.; BERGER, R. Competitividade das exportações brasileiras de móveis no mercado internacional: uma análise segundo a visão desempenho. Revista FAE, Curitiba, v. 7, n. 1, p. 51-65, 2004.

CRUZ, T. Sistemas de Informações Gerenciais. São Paulo: Atlas S.A., 2000.

FRANZONI, J. A. Formulação de estratégias de marketing internacional: um estudo das principais empresas moveleiras da região de São Bento do Sul - SC. 111 f. Dissertação (Mestrado em Engenharia Florestal) - Setor de Ciências Agrárias, Universidade Federal do Paraná, Curitiba, 2005.

GORINI, A. P. F. Panorama do setor moveleiro no Brasil, com ênfase na competitividade externa a partir do desenvolvimento da cadeia industrial de produtos sólidos de madeira. Rio de Janeiro: BNDS, 1998. 50 p. (BNDES Setorial, n, 8)

MORAES, M. A. F. D. de. Estudo da competitividade de cadeia integradas no Brasil: impactos das zonas de livre comércio. Cadeia: Madeira e móveis versão para discussão em seminários. Campinas: [UNICAMP], 2002. Nota Técnica Final. NEIT-IE-UNICAMP.

OLIVEIRA, C. G. Análise de sistemas de informação nas empresas florestais do Estado do Paraná. Dissertação (Mestrado em Engenharia Florestal) - Setor de Ciências Agrárias, Universidade Federal do Paraná, Curitiba, 1983.

OLIVEIRA, D. P. R. Sistemas de informações gerenciais. São Paulo: Atlas S.A., 2001.

REZENDE, D. A.; ABREU, A. F. Tecnologia da informação aplicada a sistemas de informações empresariais. São Paulo: Atlas S.A., 2001.

STAIR, R.M. Princípios de sistemas de informação: abordagem gerencial. Rio de Janeiro: LTC Livros Técnicos e Científicos, 1998. 